\title{
Detection of magnetic moments using a nano-SQUID: limits of resolution and sensitivity in near-field SQUID magnetometry
}

\author{
V Bouchiat \\ Institut Néel, CNRS/UJF, BP 166, F-38042 Grenoble Cedex 9, France \\ and \\ Department of Physics, Berkeley and Materials Science Division, Lawrence Berkeley National \\ Laboratory, University of California, Berkeley, CA 94720, USA \\ Received 3 September 2008, in final form 15 October 2008 \\ Published 14 May 2009 \\ Online at stacks.iop.org/SUST/22/064002
}

\begin{abstract}
We investigate the coupling efficiency of a localized magnetic moment placed at a distance $z$ from a DC-SQUID magnetometer of loop radius $a$ with nanobridges of cross section $r^{2}$. Using simple magnetostatic considerations, we show that there exist two detection regimes: the usual far-field regime $(z \gtrsim a)$ for which inductive coupling is achieved by the entire loop and a near-field regime $(z \ll a)$ where nanobridges become the active detecting elements. Simulation shows that the greatest coupling efficiency is obtained in the near-field regime $(z \ll a)$ when the magnetic moment sits directly on the nanobridge. The maximum coupling limit is given by: $1 / 2 \mu_{0} \frac{M}{r}$. Using nanoscale weak links and typical noise performance of nano-SQUID, we conclude that the limit of single molecular magnet detection can be obtained with $r \sim 1 \mathrm{~nm}$, a value reachable using carbon nanotube Josephson junctions.
\end{abstract}

(Some figures in this article are in colour only in the electronic version)

\section{Introduction}

There is growing interest to implement quantum information technologies based on the manipulation and measurement of single spins [1]. For that purpose, one needs to build a reliable and scalable magnetization measurement scheme, sensitive at the single spin level (i.e. a nanoscale single Bohr magnetometer integrated at the solid state). Among all competing magnetometry techniques [2, 3], the direct transduction from a single spin state to a measurable electrical quantity—charge, voltage or current-(a principle driving the growing field of quantum spintronics) appears to be a promising route [4], especially when combined with the recent developments in molecular electronics [5].

Today, superconducting quantum interference devices (SQUIDs) [6] provide the most sensitive magnetometers and gradiometers in the solid state. However, while these devices are extremely well suited for classical and quantum reversal studies in nanometer-sized particle and cluster magnetization measurements [7], the demonstration of a SQUID magnetometry down to the single molecule has yet to be achieved.

In a SQUID, the magnetic detection [6] is performed through inductive coupling of the loop to a local external magnetic field. Therefore, it appears clear that the device geometry has a direct influence on the magnetometry performance.

The idea of reducing the SQUID magnetometer size to improve their inductive coupling to small samples emerged some 25 years ago thanks to Ketchen et al [8]. Interestingly, the trends towards the miniaturization of nano-SQUIDs was also motivated by other very diverse objectives such as improving spatial resolution in scanning SQUID microscopy [9, 10], implementing new kinds of superconducting qubits $[11,12]$ or measuring persistent current mesoscopic normal rings [13].

Ketchen et al calculated [14] the spin sensitivity $S_{n}$ for the peculiar geometry of an assembly of spins placed at the SQUID loop center:

$$
S_{n}=\frac{2 a \Phi_{\mathrm{ns}}}{\mu_{\mathrm{B}} \mu_{0}} \text { (in number of spins), }
$$




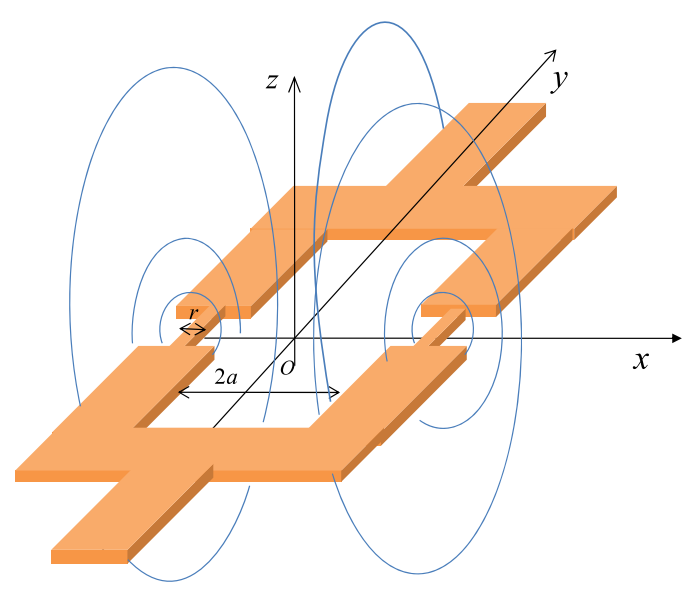

Figure 1. Schematics of a DC-SQUID based on nanobridges. It is composed of a square loop of area $4 a^{2}$ and two symmetric Dayem (lateral constrictions) bridges of section $r^{2}$. Blue lines are magnetic field lines created by the current flowing through the device.

where $\Phi_{\mathrm{ns}}$ is the SQUID flux noise, $a$ is the SQUID loop radius, $\mu_{\mathrm{B}}$ is the Bohr magneton and $\mu_{0}$ is the vacuum permeability. More recently Gallop et al $[15,16]$ investigated in detail the limits of detection of such miniaturized devices and concluded that single-spin detection $\left(S_{n}<1\right)$ could be achieved provided that the SQUID would be operated near the quantum limit. On the experimental side, the Ketchen prediction (1) has initiated a global trend towards miniaturization of the SQUID loop [15, 17-22] that showed a revival recently [23-26] in the context of the growing interest in quantum spintronics [5]. In most studies, it was concluded that, taking into account the actually measured flux noise in the best cases, the spin sensitivity $S_{n}$ in the geometry proposed by Ketchen et al [8] would be limited to values in the range of 100-1000 spins. The purpose of this paper is to explore in a quantitative way other coupling geometries, including the case where the assembly of spins sits directly on the SQUID junctions. This latter coupling geometry was already recognized as a critical improvement by Wernsdorfer et al in $1995[19,27]$ and led to important contributions to the field of molecular magnetism [28].

\section{Determination of the coupling factor between a magnetic dipole and a SQUID with nanobridge weak links}

In the following, it is considered that the assembly of spins to be detected forms a magnetic dipolar moment $M$ whose size is negligible compared to the nanobridge size and therefore can be considered as a point. It is coupled to a nano-SQUID of loop area $a^{2}$ having Dayem bridges of cross section $r^{2}$ (figure 1). The Cartesian coordinates of $M$ are denoted by $(x, y, z)$ which register the moment's position with respect to the SQUID loop center $\mathrm{O}$.

One has to calculate the coupling factor between a magnetic dipole $\vec{M}$ and the nano-SQUID. The problem can be significantly simplified by invoking the Lorentz reciprocity theorem [29] that stands for electromagnetism in a linear

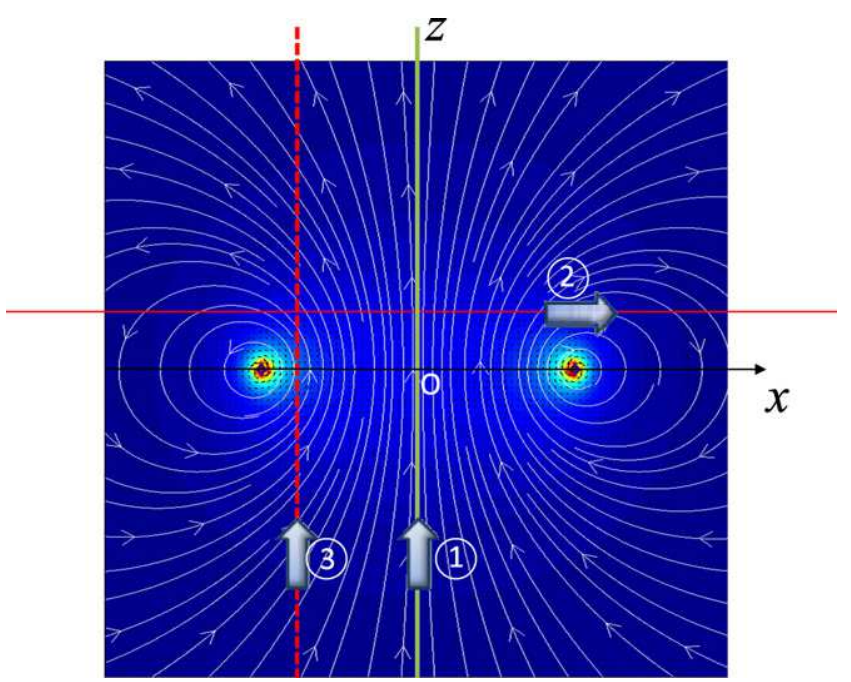

Figure 2. Cross section of the field lines create by the SQUID at plane $y=0$ showing the field concentration around the nanobridges (in red). The arrows depict the magnetic moment directions. Three cases are considered. Case (1) is a vertical moment traveling along the $z$ symmetry axis of the SQUID. Case (2) is a moment $M_{x}$ traveling along $x$ at constant altitude $z$. Case (3) is similar to case 1 but with a trajectory of equation $x=-(a-r)$ with $r \ll a$.

isotropic medium: the sources and the created fields can be interchanged. In our case, it is therefore equivalent and much simpler to consider the case of evaluating the magnetic field created by the SQUID's circular loop at the dipole position $(x, y, z)$ instead of calculating the flux generated by the dipole and threaded by the loop. This method, for example, has been previously addressed in the dual case [30] which corresponds to the single electrostatic charge sensed by a single-electron transistor acting as a sensitive electrometer. The SQUID/dipole coupling factor is therefore given by the relation: $\alpha=\frac{1}{\mu_{0} M I} \vec{M} \cdot \vec{B}$, where $B$ is the magnetic field at the dipole position created by the SQUID loop threaded by a current $I$. For the sake of simplicity, we neglect the finite strip width $w$ of the loop and we consider that a uniform and symmetric current $I$ is circulating in each arm of the SQUID. Simple magnetostatic analytical calculations are detailed in the appendix and summarized below. The coupling factor $\alpha$ can indeed be obtained in simple cases that are depicted in figure 2. We have chosen to plot the variation of $\alpha$ for a moment traveling along a specific axis. Case (1) refers to a vertical moment traveling along the SQUID symmetry axis $z$ (case including the situation considered by Ketchen [14]). Case (2) considers a horizontal moment $M_{x}$ traveling tangentially to the weak link at a constant altitude $(z=r, y=0)$. Case (3) consider a vertical moment $M_{z}$ traveling on a vertical line tangentially to the weak link $(x=a-r, y=0)$. Calculations of the spatial variation of $\alpha$ for the realistic case of a $1 \mu \mathrm{m}^{2}$ loop $(a=500 \mathrm{~nm})$ and for weak links of cross section having respective radii of $25 \mathrm{~nm}$ (size of a typical nanobridge made by state-of-the-art electron beam lithography (EBL)) and $1 \mathrm{~nm}$ (typical carbon nanotube radius) are shown in figure 3. From these curves, it appears clear that two detection regimes do exist: the usual far-field regime $(z \gtrsim a)$ for which a slightly 

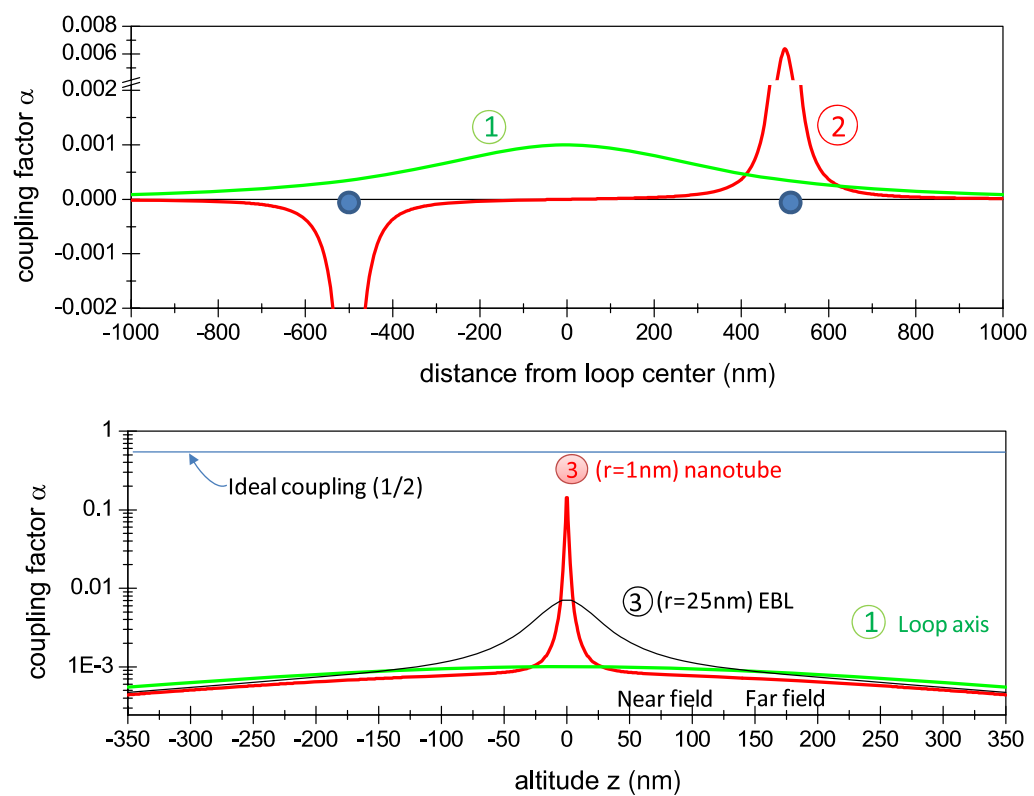

Figure 3. Spatial evolution of the coupling factor $\alpha$ for different trajectories depicted in figure 2 with realistic values $(a=500 \mathrm{~nm}$, $z=25 \mathrm{~nm}$ ). Top: comparison for trajectories of moments 1 and 2 as a function of the distance from loop center $\mathrm{O}$ showing the coupling enhancement for a magnet placed in the vicinity to one of the nanobridges (blue dots). Bottom: vertical trajectory of moments 1 (traveling along the $z$ symmetry axis) and moment 3 (traveling along $z$ with a trajectory tangential to the weak link). The simulation shows the two cases of an EBL-made nanobridge $(r=25 \mathrm{~nm})$ (black curve) and for a single-walled carbon nanotube $(r=1 \mathrm{~nm})($ red curve). A crossover of the optimal efficiency is shown between the near-field (dark centered area (red on the online version) for the nanotube case, light red area for the EBL) coupling regime $z \ll a$ and the far-field coupling regimes $r=a$ peripherical areas (in light green online). The blue line depicts the theoretical coupling limit $(\alpha=1 / 2)$ (see figure 4$)$ which is almost reached with a carbon nanotube junction.
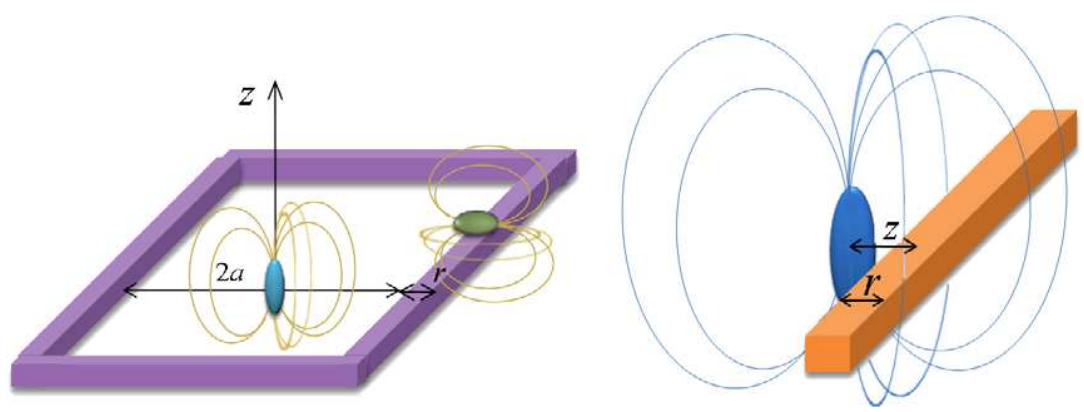

Figure 4. Left: comparison of the geometry considered by Ketchen et al and the coupling in the near-field regime. Right: schematics showing the coupling of a magnetic dipole to the nanobridge in the near-field regime. When $r, z$ are both reduced to zero, half of the flux lines are threaded by the wire, leading to a coupling limit of $1 / 2$.

better coupling is achieved when the moment is on the loop axis, as already shown in simulations of scanning SQUID microscopy $[10,31]$. More importantly, it shows a crossover from the far-field regime (clear green peripherical zones in figure 3, bottom) with loop coupling detection towards a nearfield regime $(z \ll a)$ where nanobridges themselves acts as the active detecting elements. If it remains true that maximum coupling is achieved on the loop symmetry axis in the farfield regime, it is, however, much more efficient in the nearfield regime to move the dipole off-axis and closer to the weak link since it is where the field gradient is maximum. The greatest coupling efficiency is obtained in the case for which $M$ sits directly on the nanobridge $(x=-a, y=0$, $z=0)$. In that latter case it is important to note that the narrower the weak link, the better the coupling. For the Dayem nanobridge, the coupling factor equals 0.006 while it can reach 0.3 for a nanotube bridge (in that latter geometry, however, the finite size of the molecular magnet with respect to the nanotube should give corrective terms to that simple estimation). The maximum coupling limit is then given by: $1 / 2 \mu_{0} \frac{M}{r}$ (see figure 4 , right, and appendix) giving a coupling factor of $\alpha=1 / 2$, which corresponds to the case where half the flux lines emerging from the dipole are threaded by the SQUID loop.

\section{Optimizing the SQUID coupling factor: crossover between the far-field and near-field coupling cases}

\subsection{Adapting the Ketchen formula for near-field SQUID magnetometry}

Considering the coupling study performed in the previous part, it appears that the loop field pick-up for the geometry 
considered by Ketchen et al (localized spin at loop center) [8] is rather inefficient, as most of the short range field lines emerging from the dipole are not threaded by the loop (see figure 4) and do not inductively couple to the SQUID. More precisely optimizing the SQUID near-field sensitivity implies both miniaturizing the weak link cross section and minimizing its distance to the magnetic moment to be measured. The loop size then becomes a secondary parameter as its variation essentially influences the SQUID magnetometry parameters through the change of geometric inductance. A $1 \mu \mathrm{m}^{2}$ loop appears to be sufficiently low to have a reduced inductance and enough insensitivity against far-field noise sources. Furthermore, a deep sub-micron-square loop would suffer from additional flaws as it makes the external field $B=\phi_{0} / 4 a^{2}$ required to bias the SQUID at the maximum responsivity for magnetometry detection rather intense (and eventually of the same order of magnitude of the thin film perpendicular critical field). This could, however, be partly circumvented using large superconducting pads around the SQUID loop to induce flux focusing [22, 24] but this provides a source of flux noise due to depinning of vortices.

If it is true that the loop size sets the SQUID spatial resolution in the far-field regime (for $z \gtrsim a$ ), which is the case encountered in scanning SQUID microscopy [9, 10, 31, 32], it is clear from the previous part that the sensitive part in the near-field regime $(z \ll a)$ is no longer the loop but the weak link itself.

As a consequence, for a magnetic dipole in close vicinity to a DC-SQUID weak link, the geometrical parameter (the loop radius $a$ ) presented in the original Ketchen formula (1) has to be replaced by the effective geometrical parameter $2 r / \alpha$ :

$$
S_{n}=\frac{4 r \Phi_{\mathrm{ns}}}{\alpha \mu_{\mathrm{B}} \mu_{0}} \text { (in number of spins). }
$$

\subsection{Practical implementation of nano-SQUID weak links}

This latter formula (2) is actually very good news for the ultimate sensitivity of a nano-SQUID. Indeed it is obviously much easier to miniaturize the weak link cross section $r$ than the device loop $a$. This cross section has already been scaled down to the range of tens or hundreds of $\mathrm{nm}^{2}$ using very diverse advanced nanofabrication techniques. Today two approaches are still competing and follow the complementary routes of bottom-up and top-down nanofabrication techniques.

On the one hand, weak links can be implemented using bottom-up nano-objects including semiconducting nanowires [33] or carbon nanotubes [34], or using these nanoobjects as templates for coating them with a metallic superconductor [35]. On the other hand, top-down techniques are still very promising. They mainly consist of conventional lithography techniques followed by a more advanced step mostly based on milling local probes which further reduces the weak link cross section. This has been successfully done using direct write nanolithography techniques. Several processes have been shown using focused ion beams [23-25] or using atomic force microscopy acting as a mechanical plow [36] or as a local oxidation source $[37,38]$.
Supposing that the coupling factor $\alpha$ could be made to be around unity (as is the case for carbon nanotube weak links, see figure 3 (bottom)), then the increase in sensitivity would be compatible with the detection of a single molecular magnet grafted to the carbon nanotube junction [34].

Coupling the SQUID to a small assembly of spins in the near-field regime requires local surface functionalization [39]. For that purpose, bottom-up nano-objects are the best suited as well since one can take advantage of their specific chemical reactivity [40], which is usually very different from those of the connecting superconducting electrodes.

\section{Noise parameters and fundamental limits of flux sensitivity in the near-field regime}

\subsection{Thermal dissipation of a weak link measured in DC}

For a current-biased hysteretic SQUID, thermal dissipation occurs when the weak link transits to the normal state [7]. For a typical metallic nano-weak-link having a critical current in the $1-10 \mu \mathrm{A}$ range and a normal state bridge resistance ranging between 10 and $100 \Omega$, the dissipation by Joule heating in the weak link ranges from $10 \mathrm{pW}$ to $10 \mathrm{nW}$. This power can be greatly reduced down to $10^{-16} \mathrm{~W}$ using carbon nanotube devices whose critical current can be as low as 10 pA for a normal resistance of $100 \mathrm{k} \Omega$. For a resistively shunted microbridge [41], the SQUID is continuously dissipating on the $3 \Omega$ shunt a power of $\sim 50 \mu \mathrm{V}$ which gives a $1 \mathrm{nW}$ dissipation power. Note that, by using specific control electronics $[7,20]$ that can rapidly shut down the SQUID bias once it reaches the dissipating state, the dissipation time per single measurement can be reduced to the $\mu$ s range for the metallic bridge and to the ms range for nanotube SQUIDs which gives an equivalent dissipation energy of $10^{-20}-10^{-15} \mathrm{~J}$ per single shot measurement. Finally there is hope that interfacing these weak links to microwave-based devices, such as the bifurcation amplifier $[42,43]$ that measures the effective weak link inductance rather than its critical current, a nondissipative measurement could be performed.

\subsection{SQUID back action on the magnetic moment}

In the superconducting state as well, the probing SQUID current is a source of back action on the coupled spin through the magnetic field created by the sensing current. A $1 \mu \mathrm{A}$ current circulating though the weak link generates a field of $2 \mathrm{G}$ at a distance of $1 \mathrm{~nm}$. While negligible compared to the polarization field applied during the spin manipulation, this field could induce a significant back action due to its frequency component and direction and could alter significantly the quantum properties near the magnetization reversal threshold values. Decreasing the critical current of the SQUID in the $\mathrm{nA}$ or even in the $\mathrm{pA}$ range, as demonstrated in the nanotube SQUID, provides a way to limit that back-action noise.

\subsection{Noise and detection threshold in the nanobridge SQUIDs}

It then appears clear that the original Ketchen formula which was based on the far-field calculation gave a pessimistic 

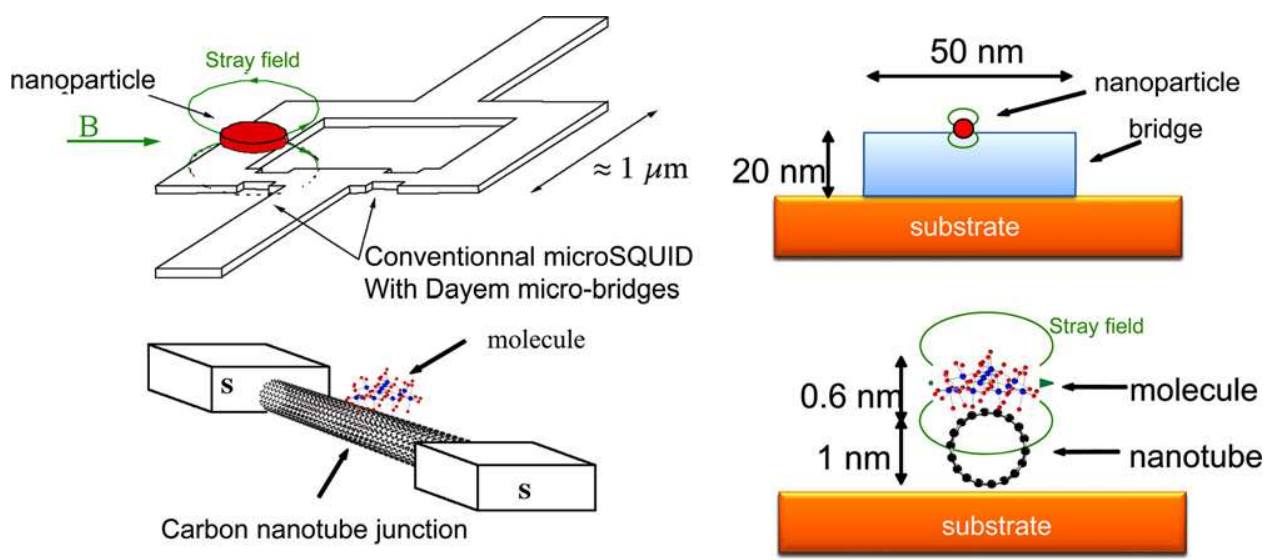

Figure 5. Practical implementation of the nano-SQUID detection in the near-field regime. Top: nanoparticle placed on a nanobridge fabricated using state-of-the-art nanolithography and thin film deposition. Bottom: molecular magnet grafted on a carbon nanotube nanobridge.

estimate of the SQUID detection threshold for nanoscale magnets.

Noise studies for SQUID-based Dayem bridges $[14,26,41,44]$ and for nanotube-based SQUIDs [34] lead to a noise $S_{\Phi}$ of $0.1-10 \mu \Phi_{0} / \mathrm{Hz}^{1 / 2}$, with the usual $1 / f$ rise at low frequency. Introducing these values in the modified formula (2) gives a SQUID sensitivity $S_{n}$ of around 1-10 Bohr magnetons, therefore validating the use of these devices to detect spin flips of 10 Bohr magnetons in amplitude.

\section{Detection of the magnetization reversal of a single molecular magnet}

Let us consider the case of a molecular magnet (taken here as a 'manganese 12' molecule) grafted to a carbon nanotube weak link (figure 5). The manganese 12 molecule is the most prominent example of a molecular magnet. It is a complex of formula $\mathrm{Mn}_{12} \mathrm{O}_{12}\left(\mathrm{MeCO}_{2}\right)_{16}\left(\mathrm{H}_{2} \mathrm{O}\right)_{4}$ 'dodecanuclear mixed valence manganese oxo-cluster with acetate ligand', it is comprised of a central $\mathrm{Mn}(\mathrm{IV})_{4} \mathrm{O}_{4}$ cube surrounded by a ring of $8 \mathrm{Mn}$ (III) units connected through bridging oxo ligands. In addition, it has 16 acetate and 4 water ligands. In $\mathrm{Mn}_{12}$, the spin state is $S=10$ since it involves 20 unpaired electrons in the ground state. At very low temperature (under a 'blocking temperature' characterized by a very slow relaxation rate) it exhibits a magneto-crystalline spin anisotropy ('Ising-type' $m= \pm S$ ). Each molecular complex then behaves as a superparamagnet with negligible intermolecular interaction. In a first approximation, the energy barrier can be written as: $U=S^{2}|D|$, where $S$ is the dimensionless total spin state and $D$ the zero-field splitting parameter. Spin-orbit coupling can be neglected. The magnetic moment created by a single $\mathrm{Mn}_{12}$ molecule is then $10 \mu_{\mathrm{B}}$. During a magnetization reversal of $\mathrm{Mn}_{12}$, the SQUID will detect a magnetic flux variation $\Delta \Phi=$ $\alpha \Delta M$, with $\Delta M \sim 2 m=20 \mu_{\mathrm{B}}$ and $\alpha$ is the geometrical molecule-SQUID coupling factor. The $\mathrm{Mn}_{12}$ molecule can be approximated by a uniformly magnetized sphere, of radius $0.5 \mathrm{~nm}$, of macro-spin $S=10$. Therefore the moment variation during magnetization reversal is $\Delta M=2 S=20 \mu_{\mathrm{B}}=$ $1.85 \times 10^{-22}$ SI. The magnetic flux variation for an $\mathrm{Mn}_{12}$ sitting on the carbon nanotube $(R=1 \mathrm{~nm})$ is given by $\Delta \Phi=$ $\alpha \mu_{0} \frac{\Delta M}{R}=0.188 \mu_{0} \frac{\Delta M}{R}=4.3 \times 10^{-20} \mathrm{~Wb}=2 \times 10^{-5} \Phi_{0}$. This value is several times below the flux noise measured in this kind of device [34].

\section{Conclusion}

A simple magnetostatic model has been presented to simulate the inductive coupling of a localized magnetic dipole to a DC nano-SQUID. The results for the coupling efficiency show a crossover between far-field detection driven by the loop geometry and a near-field detection driven by the weak links cross section. The transition between the two regimes occurs for a distance of the dipole from the SQUID center equaling the SQUID loop radius.

Optimizing the near-field inductive coupling requires both minimizing the weak link cross section and its distance to the magnetic dipole to be detected. A quantitative estimate including recent experimental results on nano-SQUIDs shows that magnetometry of single molecular magnets could be envisioned in that regime. That would allow the direct interfacing of two quantum worlds: the superconducting quantum devices and the electron spin dynamics.

\section{Acknowledgments}

The author thanks Wolfgang Wernsdorfer, John Clarke and Irfan Siddiqi for stimulating discussions. This work has been partly supported by the Miller Institute for Basic Research in Science.

\section{Appendix. Analytical calculation of the SQUID/spin coupling factor in different geometries (depicted in figures 2 and 3)}

The field along $z$ is given by the projection of the field created by the four wires corresponding to the four sides of the loop. For $z$ not greater than $a$, one can neglect the finite length of the loop sides and use the Biot and Savart formula for a field created by a infinite wire placed at a distance $r: B=\frac{\mu_{0}}{2 \pi r} I$. 
Case (1): vertical $M z$ normal to the loop moment traveling along the SQUID symmetry axis $z$ (case considered by Ketchen: see (1) in figure 2).

The resulting field corresponds to the simple formula for an on-axis field obtained using the law of Biot and Savart, integrated over a circular current loop:

$$
B_{z}=\left(\frac{\mu_{0}}{2 \pi}\right) \frac{a}{a^{2}+z^{2}} \frac{a}{\sqrt{a^{2}+z^{2}}} .
$$

Thus $\alpha=\frac{a^{2}}{z\left(\sqrt{a^{2}+z^{2}}\right)^{3}}$. The corresponding variation of $\alpha$ is shown by the red curve of figure 3 .

Case (2): a horizontal moment traveling tangentially to the weak link at a constant altitude $z=r$ (case 2 of figure 2).

The analytical expression of the off-axis field created by a circular loop has been described in detail in [45] and [46] and can be written as

$$
\begin{aligned}
& B_{x}=B_{0} \frac{1}{\pi \sqrt{Q}}\left[E(k) \frac{1-\alpha^{2}-\beta^{2}}{Q-4 \alpha}+K(k)\right] \\
& B_{\mathrm{r}}=B_{0} \frac{\gamma}{\pi \sqrt{Q}}\left[E(k) \frac{1+\alpha^{2}+\beta^{2}}{Q-4 \alpha}-K(k)\right]
\end{aligned}
$$

where $B_{x}$ is the magnetic field component that is aligned with the coil axis and $B_{\mathrm{r}}$ is the magnetic field component that is in a radial direction:

$$
\begin{array}{rlrl}
\alpha=\frac{r}{a}, & \beta=\frac{x}{a}, & \gamma & =\frac{x}{r}, \\
Q=\left[(1+\alpha)^{2}+\beta^{2}\right], & k=\sqrt{\frac{4 \alpha}{Q}}
\end{array}
$$

$K(k)$ is the complete elliptic integral function of the first kind, while $E(k)$ is the complete elliptic integral function of the second kind.

Case (3): a vertical moment normal to the loop traveling on a vertical line tangentially to the weak link $(x=a-z)$ along the line of equation $(z=a-r, y=0)$, where $r$ is the minimum distance of the dipole from the nanobridge (case 3 of figure 2). The resulting field can be written as the superposition of the field created by the two weak links (which is assimilated as a wire of infinite length) over the background field created by the SQUID loop which we consider as infinitely thin wires.

Note that one obtains at first order the same spatial dependence for a dipole $M_{x}$ parallel to the loop evolving along $z$ at constant altitude.

Direct calculation of the coupling in the near-field regime in the ideal case: magnetic dipole in direct contact to a wire of cross section $R^{2}$.

This case corresponds to the ideal coupling $(\alpha=1 / 2)$ (see figure 4 , right). The integral of flux threaded has a simple form:

$$
\begin{aligned}
\Phi & =\int \mathbf{B} \cdot \mathbf{d} \mathbf{S}=4 \int_{\gamma=-\frac{\pi}{2}}^{\frac{\pi}{2}} \int_{R}^{\infty} B_{\theta}\left(\frac{R}{\cos \gamma}\right) \frac{R \mathrm{~d} r}{\cos \gamma} \mathrm{d} \gamma \\
& =\frac{\mu_{0}}{\pi} \frac{M}{2 R} \int_{-\frac{\pi}{2}}^{\frac{\pi}{2}} \cos ^{2} \gamma \mathrm{d} \gamma=\mu_{0} \frac{M}{2 \pi R} .
\end{aligned}
$$

\section{References}

[1] Hanson R and Awschalom D D 2008 Nature 4531043

[2] Hanson R, Dobrovitski V V, Feiguin A E, Gywat O and Awschalom D D 2008 Science 320 352-5

[3] Rugar D, Budakian R, Mamin H J and Chui B W 2004 Nature 430329

[4] Wolf S A, Awshalom D D, Buhrman R A, Daughton J M, von Molnàr S, Roukes M L, Chtchelkanova A Y and Treger D M 2001 Science 2941488

[5] Bogani L and Wernsdorfer W 2008 Nat. Mater. 7 179-86

[6] Clarke J and Braginski A I 2004 The SQUID Handbook (Weinheim: Wiley-VCH)

[7] Wernsdorfer W 2001 Adv. Chem. Phys. 188 99-190

[8] Ketchen M B, Kopley T and Ling H 1984 Appl. Phys. Lett. 44 1008-10

[9] Kirtley J R, Ketchen M B, Stawiasz K G, Sun J Z, Gallagher W J, Blanton S H and Wind S J 1995 Appl. Phys. Lett. 661138

[10] Hasselbach K, Veauvy C and Mailly D 2000 Physica C 332 140-7

[11] Mooij J E, Orlando T P, Levitov L, Tian L, van der Wal C and Lloyd S 1999 Science 2851036

[12] Chiorescu I, Nakamura Y, Harmans C J P M and Mooij J E 2003 Science 2991869

[13] Rabaud W, Saminadayar L, Mailly D, Hasselbach K, Benoît A and Etienne B 2001 Phys. Rev. Lett. 863124

[14] Ketchen M B, Awschalom D D, Gallagher W J, Kleinsasser A W, Sandstrom R L, Rozen J R and Bumble B 1989 IEEE Trans. Magn. 251212

[15] Gallop J C, Josephs-Franks P W, Davies J, Hao L and Macfarlane J C 2002 Physica C 368109

[16] Gallop J 2003 Supercond. Sci. Technol. 161575

[17] Ketchen M B 1993 IEEE Trans. Appl. Supercond. 32586

[18] Mailly D, Chapelier C and Benoit A 1993 Phys. Rev. Lett. 702020

[19] Wernsdorfer W, Hasselbach K, Mailly D, Barbara B, Benoit A, Thomas L and Suran G 1995 J. Magn. Magn. Mater. 14533

[20] Wernsdorfer W 1996 PhD Thesis J Fourier University Grenoble

[21] Josephs-Franks P W, Reed R P and Pakes C I 2000 Physica C 280540

[22] Lam S K H and Tilbrook D L 2003 Appl. Phys. Lett. 82 1078-80

[23] Tettamanzi G C, Pakes C I, Potenza A, Marrows C H, Prawer S and Jamieson D N 2006 30th Annual Condensed Matter and Materials Mtg (Australia)

[24] Troeman A G P, Derking H, Borger B, Pleikies J, Veldhuis D and Hilgenkamp H 2007 Nano Lett. 7 2152-6

[25] Hao L, Macfarlane J C, Gallop J C, Cox D, Beyer J, Drung D and Schurig T 2008 Appl. Phys. Lett. 92192507

[26] Granata C, Esposito E, Vettoliere A, Petti L and Russo M 2008 Nanotechnology 19275501

[27] Wernsdorfer W et al 1997 Phys. Rev. Lett. 79 4014-7

[28] Gatteschi D, Sessoli R and Villain J 2006 Molecular Nanomagnets (Oxford: Oxford University Press)

[29] Lorentz H A 1896 Versl. Kon. Akad. Wentensch. 4 p 176; also in P Zeeman and A D Fokker (ed) 1936 H A Lorentz, Collected Papers (The Hague, Holland: Martinus Nijhoff) vol III pp 1-11

[30] Bouchiat V, Chardin G, Devoret M H and Esteve D 1997 Hyperfine Interact. 109345

[31] Chatraphorn S, Fleet E F and Wellstood F C 2002 J. Appl. Phys. 924731

[32] Bradley J R, Nestor G S and Wikswo J P Jr 1989 J. Appl. Phys. 65 361-72

[33] van Dam J A, Nazarov Y V, Bakkers E P, De Franceschi S and Kouwenhoven L P 2006 Nature 442667

[34] Cleuziou J-P, Wernsdorfer W, Bouchiat V, Ondarcuhu T and Monthioux M 2006 Nat. Nanotechnol. 153 
[35] Hopkins D, Pekker D, Goldbart P and Bezryadin A 2005 Science 3081762

[36] Irmer B, Blick R H, Simmel F, Godel W, Lorenz H and Kotthaus J P 1998 Appl. Phys. Lett. 73 2051-3

[37] Bouchiat V, Faucher M, Thirion C, Wernsdorfer W, Fournier T and Pannetier B 2001 Appl. Phys. Lett. 79 123-5

[38] Faucher M, Fournier T, Pannetier B, Thirion C, Wernsdorfer W, Villegier J C and Bouchiat V 2002 Physica C 368211

[39] Lam S K H, Yang W, Wiogo H T R and Foley C P 2008 Nanotechnology 19285303

[40] Besteman K, Lee J O, Wiertz F G M, Heering H A and Dekker C 2003 Nano Lett. 3 727-30
[41] Lam S K H 2006 Supercond. Sci. Technol. 19963

[42] Siddiqi I et al 2005 Phys. Rev. Lett. 94027005

[43] Siddiqi I et al 2004 Phys. Rev. Lett. 93207002

[44] Hao L, Macfarlane J C, Gallop J C, Cox D, Beyer J, Drung D and Schurig T 2008 Appl. Phys. Lett. 92192507

[45] Montgomery D B and Terrell J 1961 Some Useful Information for the Design of Air-Core Solenoids (Cambridge: Massachusetts Inst of Tech Francis Bitter National Magnet Lab)

[46] Smythe W R 1950 Static and Dynamic Electricity (New York: McGraw-Hill) 